\title{
Somatotyping and Some Physical Characteristics of Trained Male and Female Young Table Tennis Players
}

\author{
Pritha Chatterjee, Arush Goswami, Anupam Bandyopadhyay* \\ Department of Physiology, Serampore College, University of Calcutta, West Bengal, India
}

Email address:

bando_anupam@yahoo.co.in (A. Bandyopadhyay)

\section{To cite this article:}

Pritha Chatterjee, Arush Goswami, Anupam Bandyopadhyay. Somatotyping and Some Physical Characteristics of Trained Male and Female Young Table Tennis Players. American Journal of Sports Science. Special Issue: Kinanthropometry. Vol. 4, No. 1-1, 2016, pp. 15-21. doi: $10.11648 /$ j.ajss.s.2016040101.13

\begin{abstract}
The aim of this study was to analyze the relationship among somatotype characteristics and physical training of young male and female Indian table tennis players. A total of 29 players (14 males and 15 females), who represented district, state and national level competitions, were evaluated with the sex as a factor. Different body measurements were recorded following the guidelines proposed by the ISAK such as body mass, height, skinfold thicknesses at different sites (biceps, triceps, subscapular, supraspinale and medial calf), girths (arm relaxed, thigh, and calf) and breadths (biepicondylar humerus and femur). In addition to anthropometrical analysis, body composition and somatotype of the participants had been assessed. A t- test for independent samples was performed to examine the statistical differences between sex groups and a Pearson's coefficient was applied to evaluate the correlation between variables. An endomorph-ectomorph somatotype characteristics was observed for the entire group. Analysis, taking into account the sex factor, revealed an endomorph-mesomorph somatotype for the males and an endomorph-ectomorph somatotype for the females. Data corresponding to body composition contrasted by sex showed higher body fat percentage for the female group than the males. Within the tested age interval range, body fat content in female players was higher than the male counterparts. Although these differences might be the consequence of a normal growth, it was advisable to integrate educational and nutritional strategies in order to maintain an optimum body fat content. Training procedure must be considered to improve the body type for the specific sports event.
\end{abstract}

Keywords: Table Tennis, Body Composition, Somatotype

\section{Introduction}

Table Tennis is an individual asymmetric sport, in which hits are required with great speed and power. Technical actions that take place during the game are unilateral at the trunk and upper extremities level. Thus the dominant side of the player goes into action repeatedly and exclusively. It is an acyclic sport in which work and rest time periods are continuously alternated. Also, the intensity developed during the match makes it mixed activity, taking into account the energetic metabolism [1]. Table Tennis is a game characterized by consecutive series of fast and powerful hits against a lightweight ball. This has an alternating demand of aerobic-anaerobic requirement, caused by short and intermittent exercise and efforts with incomplete recoveries. Moreover, the dominant arm is totally involved with different positions that link the racket-arm to develop techniques of this sport. The most important physical capacities of the players are endurance and velocity, due to short time high intensity periods under anaerobic metabolism that characterize the match: Players need velocity training while the capacity to face match duration mainly depends of endurance training [2]. However, strength, coordination and flexibility may also have a key role in this sport [3]. It is evident that the physical training is an important factor to reach sport success; several studies demonstrated that, at the same training level, the best performances are obtained by athletes with more compatible anatomic conditions [4].

Since 1940 numerous studies on body composition influenced the development of somatotyping [5] and, in the last 20 years, many studies had defined the somatotype profile of athletes in different sports. Research interest in anthropometric characteristics and body composition of the players of different sports had been developed during the last decades. Different studies supported the requirement of specific anthropometric characteristics which determine the suitability of the player for best performance in that specific 
game $[6,7]$. Athletic performance is, to a large degree, dependent on the athlete's ability to sustain power (both anaerobically and aerobically) and to overcome resistance, or drag. Both of these factors are interrelated with the athlete's body composition. Coupled with the common perception of many athletes who compete in sports where appearance is a concern for the athlete and the common perception of these athletes (swimming, diving, gymnastics, and figure skating), attainment of an ideal body composition often becomes a central theme of training. Besides the aesthetic and performance reasons for wanting to achieve an optimal body composition, there may also be safety reasons. These structural relationships have been studied through observational investigations revealing how athletes with the same sport performances presented similar anthropometric parameters and body composition. Within specific sports, sport disciplines or playing positions in team sport games, it is evident that an almost identical somatotype profile dominates, characterised by certain anthropometric features that are crucial or at least extremely important for achieving top competitive results. Many authors had attempted to establish the relationship between somatotype and competitive success or performance levels in different sports $[8,9,10,11,12]$ and it would be remarkable to establish whether and to what extent the different somatotypes themselves influence the competitive success of juvenile Table Tennis players and to determine the importance of physique in achieving good competitive results in table tennis. The physique of an athlete is the configuration or build of the entire body, and the assessment of the physique is most often expressed in the context of somatotype [13].Somatotype is a composite of the contributions of three components: endomorphy (relative fatness), mesomorphy (relative musculoskeletal robustness), and ectomorphy (relative linearity) $[13,14,15]$.

\section{Aim and Objectives}

The main objectives of the present study was to explore the possible role of training in 10-20 years aged male and female right handed Table Tennis players. The objectives of the present study were aimed at following points:

1. To compare the effects of similar type of training in male \& female Table Tennis players in similar age ranges.

2. How bodytype of the players can be improved by improving the physical training methods.

3. Whether the bodytype of the both male and female Table Tennis players affect their performances.

\section{Method}

\subsection{Subjects}

In the present study male right handed Table Tennis players $(n=14)$ who were trained for 2 to 8 years with an average age of $13.29( \pm 1.86)$ years and female right handed Table Tennis players $(n=15)$ who were trained for 4 to 12 years with an average age of $13.93( \pm 3.13)$ years participated as subjects. Individual NFHS (National Standard of Living Index) and SCAT (Sports Competition Anxiety Test) were carried out in each subject. Participants of this study were from Hooghly district, West Bengal, India with participation experiences at district level, State and National level competitions. All the players were involved in regular scheduled training programs of $30 \mathrm{~min}$ free hand exercises followed by 90 to $120 \mathrm{~min}$ game practice with full efforts intermittently for 6 days per week. All the participants were students and do not participate in any other recreational game. All measurements were conducted at club premises and in departmental laboratory in the month of March, 2015. Consents were taken from each participant along with their parents and club authorities. The study had an ethical permission from the Institutional Human Ethics Committee and ethical parts were followed accordingly while conducting the tests.

\subsection{Anthropometric Measurements}

Anthropometric measurements were done on same day for each subject in same session to avoid technical error. Level 1 Anthropometrists accredited by International Society for the Advancement of Kinanthropometry (ISAK) [16] was involved in the measurements. Methods described in the ISAK manual [17] were followed. Stature was measured with an Anthropometric Rod up to $1 \mathrm{~mm}$ and body mass was measured with an electronic weighing machine. Skinfold thicknesses were measured with a Slim guide skinfold caliper (CESCORF). Anthropometric tape and sliding caliper (CESCORF) were used to measure circumferences and bone diameter respectively.

\subsection{Somatotype}

Heath - Carter [18] method was followed for somatotype rating. The following equations were uses for calculating somatotype.

Endomorphy $=-0.7182+0.1451 \times \Sigma \mathrm{SF}-0.00068 \times$ $\Sigma \mathrm{SF}^{2}+0.0000014 \times \Sigma \mathrm{SF}^{3}$ where $\Sigma \mathrm{SF}=$ (sum of triceps, subscapular and supraspinale skinfolds) multiplied by (170.18/height in $\mathrm{cm})$.

Mesomorphy $=0.858 \times$ humerus breadth $+0.601 \times$ femur breadth $+0.188 \times$ corrected arm girth $+0.161 \times$ corrected calf girth - height $\times 0.131+4.5$.

Three different equations are used to calculate ectomorphy according to the height -weight ratio (HWR):

If HWR is greater than or equal to 40.75 then, Ectomorphy $=0.732 \times \mathrm{HWR}-28.58$.

If HWR is less than 40.75 and greater than 38.25 then,Ectomorphy $=0.463 \times$ HWR -17.63 .

If HWR is equal to or less than 38.25 then, Ectomorphy = 0.1 .

\subsection{Body Fat \%}

Durnin and Womersley [19] technique was followed for body density. Body fat $\%$ was derived from the equation of Brozek et al [20]. 


\subsection{Physical Performances}

Flexibility tests (lower back \& hamstring flexibility, ankle flexibility, trunk \& neck flexibility, shoulder flexibility \& elastic leg strength) were performed $\&$ agility of the players of both groups was also measured.

\subsection{Statistical Analyses}

Mean values \& Standard Deviation of each mentioned parameters of both sexes were calculated. The unpaired two tail T-test was done to compare each of the parameters of the both genders, Probability of error due to random sampling was rejected at the level of $p<0.05$. The correlation ( $r$ ) is done for all parameters with Body height $(\mathrm{cm})$, Body weight $(\mathrm{kg})$, Fat percentage (\%), Hand span of both hands (cm), Arm girth (cm), Calf girth $(\mathrm{cm})$, Biepicondylar humerus circumference $(\mathrm{cm})$, Biepicondylar femur circumference $(\mathrm{cm})$, Chest circumference $(\mathrm{cm})$, Waist circumference $(\mathrm{cm})$, Hip circumference $(\mathrm{cm})$, Mid thigh circumference $(\mathrm{cm}) \&$ Upper thigh circumference $(\mathrm{cm})$. by Pearson's Correlation Coefficient (r).

\section{Result}

The mean value, standard deviation and level of significance of Height $(\mathrm{cm})$, Weight $(\mathrm{kg})$, Body Fat \%, Total fat content $(\mathrm{kg})$, Lean body mass $(\mathrm{kg})$, Lower back \& Hamstring flexibility $(\mathrm{cm})$, Ankle flexibility $(\mathrm{cm})$, Trunk \& Neck flexibility $(\mathrm{cm})$, Shoulder flexibility $(\mathrm{cm})$, Elastic leg strength (cm), Agility (sec), Hand span of both hands (cm), Handgrip strength of both hands $(\mathrm{kg})$, Arm girth $(\mathrm{cm})$, Calf girth $(\mathrm{cm})$, Biepicondylar humerus circumference $(\mathrm{cm})$, Biepicondylar femur circumference $(\mathrm{cm})$, Chest circumference $(\mathrm{cm})$, Waist circumference $(\mathrm{cm})$, Hip circumference $(\mathrm{cm})$, Mid thigh circumference $(\mathrm{cm})$ \&Upper thigh circumference $(\mathrm{cm})$ of male $(n=14) \&$ female $(n=15)$ trained table tennis players are shown in Table-1. In Table-2 and Table 3, the correlation values \& level of significance of Height $(\mathrm{cm})$, Weight $(\mathrm{kg})$, Hand span $(\mathrm{cm})$, Arm girth $(\mathrm{cm})$, Calf girth $(\mathrm{cm})$, Biepicondylar humerus circumference (mm), Biepicondylar femur circumference $(\mathrm{mm})$, Chest circumference $(\mathrm{cm})$, Waist circumference $(\mathrm{cm})$, Hip circumference $(\mathrm{cm})$, Mid thigh circumference $(\mathrm{cm}) \&$ Upper thigh circumference $(\mathrm{cm})$ of both male $\&$ female trained table tennis players are represented.

Table 1. Anthropometric characteristics, physical performances and motor abilities of male and female Table Tennis players of India $(n=$ Sample size ; $p=$ Probability of error due to random sampling).

\begin{tabular}{|c|c|c|c|c|}
\hline \multicolumn{2}{|l|}{ Parameters } & Male $(n=14)$ Mean \pm SD & Female $(n=15)$ Mean \pm SD & Level of Significance \\
\hline \multicolumn{2}{|l|}{ Height (cm) } & $155.82 \pm 14.24$ & $149.39 \pm 7.85$ & Not Significant \\
\hline \multicolumn{2}{|l|}{ Weight (kg) } & $47.64 \pm 10.31$ & $47.80 \pm 19.23$ & Not Significant \\
\hline \multicolumn{2}{|l|}{ Body Fat \% } & $16.43 \pm 4.96$ & $23.86 \pm 8.54$ & $\mathrm{p}<0.05$, Significant \\
\hline \multicolumn{2}{|l|}{ Total fat content $(\mathrm{kg})$} & $8.48 \pm 2.54$ & $12.40 \pm 4.44$ & $\mathrm{p}<0.05$, Significant \\
\hline \multicolumn{2}{|l|}{ Lean body mass (kg) } & $43.38 \pm 2.69$ & $39.60 \pm 4.44$ & $\mathrm{p}<0.05$, Significant \\
\hline \multicolumn{2}{|c|}{ Lower back \& Hamstring flexibility $(\mathrm{cm})$} & $25.31 \pm 6.15$ & $26.88 \pm 5.30$ & Not Significant \\
\hline \multicolumn{2}{|l|}{ Ankle flexibility $(\mathrm{cm})$} & $77.64 \pm 13.10$ & $70.33 \pm 12.33$ & Not Significant \\
\hline \multicolumn{2}{|c|}{ Trunk \& Neck flexibility (cm) } & $49.63 \pm 10.12$ & $51.77 \pm 8.08$ & Not Significant \\
\hline \multicolumn{2}{|l|}{ Shoulder flexibility $(\mathrm{cm})$} & $16.53 \pm 12.29$ & $23.30 \pm 13.20$ & Not Significant \\
\hline \multicolumn{2}{|l|}{ Elastic leg strength (cm) } & $36.74 \pm 13.04$ & $28.42 \pm 7.65$ & $\mathrm{p}<0.05$, Significant \\
\hline \multicolumn{2}{|l|}{ Agility (sec) } & $11.6 \pm 0.92$ & $11.90 \pm 1.51$ & Not Significant \\
\hline \multirow{2}{*}{ Hand span $(\mathrm{cm})$} & Right & $19.14 \pm 4.13$ & $18.85 \pm 1.66$ & Not Significant \\
\hline & Left & $19.33 \pm 4.36$ & $18.95 \pm 1.38$ & Not Significant \\
\hline \multirow{2}{*}{ Handgrip strength (kg) } & Right & $17.14 \pm 10.47$ & $11.93 \pm 7.27$ & Not Significant \\
\hline & Left & $13.64 \pm 9.52$ & $10.60 \pm 7.61$ & Not Significant \\
\hline \multicolumn{2}{|l|}{ Arm girth (cm) } & $24.39 \pm 2.40$ & $23.06 \pm 5.05$ & Not Significant \\
\hline \multicolumn{2}{|l|}{ Calf girth $(\mathrm{cm})$} & $31.76 \pm 2.40$ & $31.57 \pm 5.55$ & Not Significant \\
\hline \multicolumn{2}{|c|}{ Biepicondylar humerus breath $(\mathrm{cm})$} & $5.81 \pm 0.62$ & $5.40 \pm 0.35$ & $\mathrm{p}<0.05$, Significant \\
\hline \multicolumn{2}{|c|}{ Biepicondylar femur breadth $(\mathrm{cm})$} & $8.97 \pm 0.75$ & $8.21 \pm 0.86$ & $\mathrm{p}<0.05$, Significant \\
\hline \multicolumn{2}{|l|}{ Chest circumference $(\mathrm{cm})$} & $79.36 \pm 7.79$ & $79.99 \pm 16.39$ & Not Significant \\
\hline \multicolumn{2}{|l|}{ Waist circumference $(\mathrm{cm})$} & $72.86 \pm 9.01$ & $69.08 \pm 15.29$ & Not Significant \\
\hline \multicolumn{2}{|l|}{ Hip circumference $(\mathrm{cm})$} & $81.68 \pm 6.80$ & $86.53 \pm 15.02$ & Not Significant \\
\hline \multicolumn{2}{|c|}{ Mid thigh circumference $(\mathrm{cm})$} & $47.36 \pm 4.32$ & $44.67 \pm 8.01$ & Not Significant \\
\hline \multicolumn{2}{|c|}{ Upper thigh circumference $(\mathrm{cm})$} & $50.04 \pm 4.71$ & $50.70 \pm 10.46$ & Not Significant \\
\hline
\end{tabular}

No statistically significant differences were observed in male and female trained right handed Tennis players in Height $(\mathrm{cm})$, Weight $(\mathrm{kg})$, Lower back \& Hamstring flexibility $(\mathrm{cm})$, Ankle flexibility $(\mathrm{cm})$, Trunk \& Neck flexibility $(\mathrm{cm})$, Shoulder flexibility $(\mathrm{cm})$, Agility ( $\mathrm{sec}$ ), Hand span of both hands $(\mathrm{cm})$, Handgrip strength of both hands $(\mathrm{kg})$, Arm girth $(\mathrm{cm})$, Calf girth $(\mathrm{cm})$, Chest circumference $(\mathrm{cm})$, Waist circumference $(\mathrm{cm})$, Hip circumference $(\mathrm{cm})$, Mid thigh circumference $(\mathrm{cm}) \& U$ pper thigh circumference $(\mathrm{cm})$ (Table 1$)$. Body fat percentage and total fat content is significantly higher $(p<0.05)$ in female than their male counterparts (Table 1), but lean body mass was much higher in male than female players and it was statistically significant $(p<0.05)$ (Table 1). Elastic leg strength was found to be higher in male players than female players and it was statistically significant $(\mathrm{p}<0.05)$ (Table 1$)$ Shoulder flexibility of male players were significantly higher than the female players at $p<0.05$ level. Both biepicondylar humerus breadth and biepicondylar femur breadth were slightly higher in male Table Tennis players 
than their female counterparts and they were statistically $(\mathrm{p}<0.05)$ significant (Table 1$)$.

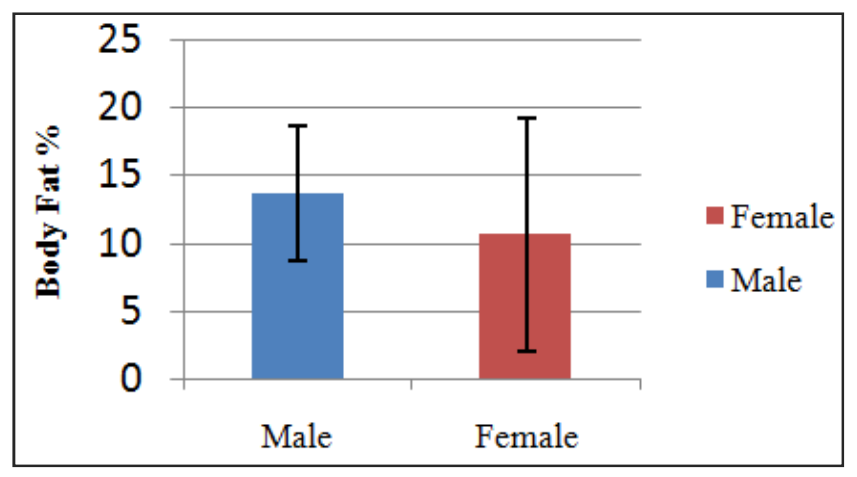

Figure 1. Mean \pm Sd Values of Body fat \% of Trained Male \& Female Table tennis Players.

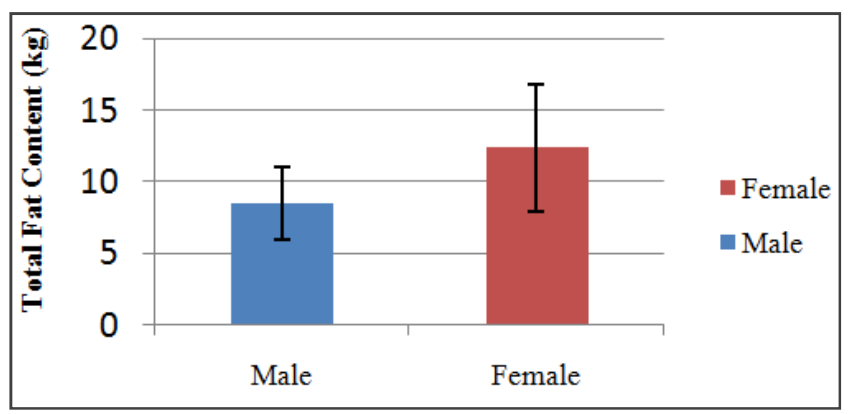

Figure 2. Mean \pm Sd Values of Lean Total Fat Content ( $\mathrm{kg}$ ) of Trained Male \& Female Table tennis Players.

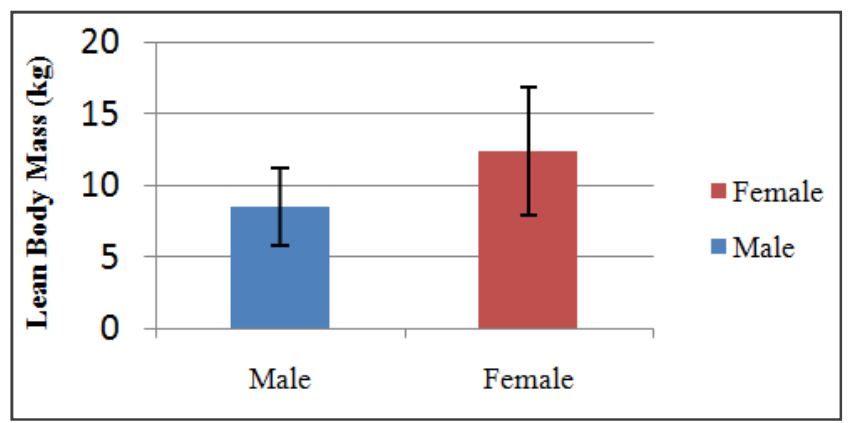

Figure 3. Mean \pm Sd Values of Lean Body Mass $(\mathrm{kg})$ of Trained Male \& Female Table tennis Players.

In Table 2 and Table 3, the positive correlations were found between height and weight of both male and female Table Tennis players and they were statistically $(p<0.05)$ significant. Hand span of right hand was positively correlated with height in both genders but hand span of left hand of female players negatively correlated with height. Height positively correlated with calf girth in both male and female players and it was significant $(p<0.05)$. Surprisingly, there is a significant correlation between height and arm girth in male but not in female subjects. Biepicondylar humerus breadth significantly correlated with height when male Table Tennis players were considered, but biepicondylar femur breadth significantly $(\mathrm{p}<0.05)$ correlated with height $(\mathrm{cm})$ in both genders. Chest circumference and waist circumference were insignificantly correlated with height in male, but those were significant in female players. Both in male and female, hip and mid thigh circumferences were significantly $(p<0.05)$ correlated with height, but upper thigh circumference was correlated with height which was seen only in female. Weight significantly $(p<0.05)$ correlated with hand span of both hands, arm girth and calf girth in male, but weight was significantly $(p<0.05)$ correlated with biepicondylar femur breadth, chest circumference, waist circumference, hip circumference, mid thigh circumference and upper thigh circumference in both male and female. Negative correlations coefficient was found between body fat $\%$ and hand span of female Table Tennis players. Body fat $\%$ was significantly $(p<0.05)$ correlated with arm girth, calf girth, biepicondylar humerus breadth, biepicondylar femur breadth, chest circumference, hip circumference, mid thigh circumference and upper thigh circumference in female only, but body fat $\%$ was significantly $(p<0.05)$ correlated with waist circumference, both in male and female. There were significant correlationships observed while correlated left hand span and hip circumference with right hand span in both male and female, but calf girth, chest circumference, waist circumference, mid thigh and upper thigh circumference were significantly $(\mathrm{p}<0.05)$ correlated with right hand span only in female players. Left hand span was positively correlated with arm girth, biepicondylar humerus breadth, chest circumference, waist circumference and upper thigh circumference only in female, but left hand span was positively correlated with mid thigh circumference in both genders and with biepicondylar femur circumference in male. Arm girth was significantly $(p<0.05)$ correlated with biepicondylar femur breadth, chest circumference, waist circumference, hip circumference, mid thigh circumference and upper thigh circumference in both male and female players, but insignificant with biepicondylar humerus breadth. Calf girth was significantly $(p<0.05)$ correlated with biepicondylar femur breadth, chest circumference, waist circumference, hip circumference, mid thigh circumference and upper thigh circumference in both male and female players, but insignificant with biepicondylar humerus breadth in female but significant in male. Biepicondylar humerus breadth was positively correlated with biepicondylar femur breadth, hip circumference and mid thigh circumference in both sexes, but it was significantly $(p<0.05)$ correlated with waist circumference and upper thigh circumference of the female players. Biepicondylar femur breadth was very much significantly $(p<0.05)$ correlated with chest circumference, hip circumference, mid thigh circumference and upper thigh circumference in both male and female players but it was insignificant with waist circumference in male, and significant $(\mathrm{p}<0.05)$ in female. There was significant $(p<0.05)$ correlation between chest circumference and hip circumference, mid thigh circumference, upper thigh circumference in both male and female players. Positive and significant $(p<0.05)$ correlationships were found between waist circumference and hip, mid thigh and upper thigh circumferences in both sexes. Hip circumference was 
significantly $(p<0.05)$ correlated with mid thigh and upper thigh circumferences in both male and female Table Tennis players. Mid thigh circumference was significantly $(\mathrm{p}<0.05)$ correlated with upper thigh circumference.

Table 2. The correlation values of Height (cm), Weight $(\mathrm{kg})$, Hand span (cm), Arm girth (cm), Calf girth (cm), Biepicondylar humerus circumference (mm), Biepicondylar femur circumference (mm), Chest circumference (cm), Waist circumference (cm), Hip circumference (cm), Mid thigh circumference (cm) \& Upper thigh circumference (cm).

\begin{tabular}{|c|c|c|}
\hline Parameters & Male (n=14) & Female $(n=15)$ \\
\hline Height vs Hand span (cm) & $0.753, \mathrm{p}<0.05$, Significant & $0.677, \mathrm{p}<0.05$, Significant \\
\hline Height $(\mathrm{cm})$ vs Calf girth $(\mathrm{cm})$ & $0.691, \mathrm{p}<0.05$, Significant & $0.816, \mathrm{p}<0.05$,Significant \\
\hline Height $(\mathrm{cm})$ vs Biepicondylar humerus circumference $(\mathrm{cm})$ & $0.731, \mathrm{p}<0.05$, Significant & 0.386 , Not Significant \\
\hline Height $(\mathrm{cm})$ vs Biepicondylar femur circumference $(\mathrm{mm})$ & $0.881, \mathrm{p}<0.05$, Significant & $0.550, \mathrm{p}<0.05$, Significant \\
\hline Height $(\mathrm{cm})$ vs Hip circumference $(\mathrm{cm})$ & $0.725, \mathrm{p}<0.05$, Significant & $0.802, \mathrm{p}<0.05$,Significant \\
\hline Height $(\mathrm{cm})$ vs Mid thigh circumference $(\mathrm{cm})$ & $0.622, \mathrm{p}<0.05$, Significant & $0.827, \mathrm{p}<0.05$,Significant \\
\hline Weight (kg) vs Arm girth (cm) & $0.813, \mathrm{p}<0.05$, Significant & $0.959, \mathrm{p}<0.05$, Significant \\
\hline Weight (kg) vs Calf girth (cm) & $0.916, \mathrm{p}<0.05$, Significant & $0.972, \mathrm{p}<0.05$,Significant \\
\hline Weight $(\mathrm{kg})$ vs Biepicondylar humerus breadth $(\mathrm{cm})$ & $0.730, \mathrm{p}<0.05$, Significant & 0.590, Not Significant \\
\hline Weight (kg) vs Biepicondylar femur breadth (cm) & $0.933, \mathrm{p}<0.05$, Significant & $0.852, \mathrm{p}<0.05$,Significant \\
\hline Weight (kg) vs Chest circumference (cm) & $0.852, \mathrm{p}<0.05$, Significant & $0.985, \mathrm{p}<0.05$,Significant \\
\hline Weight (kg) vs Waist circumference $(\mathrm{cm})$ & $0.624, \mathrm{p}<0.05$, Significant & $0.986, \mathrm{p}<0.05$, Significant \\
\hline Weight $(\mathrm{kg})$ vs Hip circumference $(\mathrm{cm})$ & $0.958, \mathrm{p}<0.05$, Significant & $0.981, \mathrm{p}<0.05$,Significant \\
\hline Weight $(\mathrm{kg})$ vs Mid thigh circumference $(\mathrm{cm})$ & $0.877, \mathrm{p}<0.05$, Significant & $0.969, \mathrm{p}<0.05$, Significant \\
\hline Weight $(\mathrm{kg})$ vs Upper thigh circumference $(\mathrm{cm})$ & $0.862, \mathrm{p}<0.05$, Significant & $0.979, \mathrm{p}<0.05$,Significant \\
\hline
\end{tabular}

Table 3. Correlation values of Hand span left (cm),Biepicondylar humerus circumference (mm), Biepicondylar femur circumference (mm), Arm girth (cm), Calf girth (cm), Upper thigh circumference (cm), Mid thigh circumference (cm). $n=$ Sample size; $p=$ Probability of error due to random sampling.

\begin{tabular}{|c|c|c|}
\hline Hand span Left $(\mathrm{cm})$ vs Biepicondylar humerus circumference $(\mathrm{mm})$ & 0.356647, Not Significant & $0.622, \mathrm{p}<0.05$,Significant \\
\hline Hand span (cm) Left vs Biepicondylar femur circumference (mm) & $0.680, \mathrm{p}<0.05$, Significant & 0.387, Not Significant \\
\hline Arm girth $(\mathrm{cm})$ vs Calf girth $(\mathrm{cm})$ & $0.763, \mathrm{p}<0.05$, Significant & $0.932, \mathrm{p}<0.05$,Significant \\
\hline Arm girth (cm) vs Biepicondylar humerus circumference (mm) & 0.381, Not Significant & 0.530 , Not Significant \\
\hline Calf girth (cm) vs Biepicondylar humerus circumference (mm) & $0.802, \mathrm{p}<0.05$, Significant & 0.492, Not Significant \\
\hline Calf girth $(\mathrm{cm})$ vs Biepicondylar femur circumference $(\mathrm{mm})$ & $0.838, \mathrm{p}<0.05$, Significant & $0.783, \mathrm{p}<0.05$,Significant \\
\hline Calf girth $(\mathrm{cm})$ vs Mid thigh circumference $(\mathrm{cm})$ & $0.845, \mathrm{p}<0.05$, Significant & $0.959, \mathrm{p}<0.05$,Significant \\
\hline Biepicondylar femur circumference $(\mathrm{mm})$ vs Mid thigh circumference $(\mathrm{cm})$ & $0.776, \mathrm{p}<0.05$, Significant & $0.801, \mathrm{p}<0.05$,Significant \\
\hline Biepicondylar femur circumference (mm) vs Upper thigh circumference $(\mathrm{cm})$ & $0.741, \mathrm{p}<0.05$, Significant & $0.784, \mathrm{p}<0.05$, Significant \\
\hline
\end{tabular}

Table 4. Somatotype categories of trained young male and female table tennis players.

\begin{tabular}{|c|c|c|c|}
\hline \multicolumn{2}{|l|}{ Male $(n=14)$} & \multicolumn{2}{|l|}{ Female $(n=15)$} \\
\hline Endo-Meso-Ecto & Category & Endo-Meso-Ecto & Category \\
\hline 7.1-4.9-0.9 & MESOMORPHIC ENDOMORPH & $3.1-2.5-4.1$ & ENDOMORPHIC ECTOMORPH \\
\hline $5.5-4.7-1.6$ & MESOMORPHIC ENDOMORPH & $3.2-2.7-4.2$ & ENDOMORPHIC ECTOMORPH \\
\hline $4.6-4.0-3.2$ & MESOMORPHIC ENDOMORPH & $1.6-2.5-4.7$ & MESOMORPHIC ECTOMORPH \\
\hline $3.8-3.4-3.9$ & ENDOMORPHIC ECTOMORPH & $1.2-2.1-5.8$ & MESOMORPHIC ECTOMORPH \\
\hline $4.6-3.5-3.6$ & ECTOMORPHIC ENDOMORPH & $5.6-3.6-1.8$ & MESOMRPHIC ENDOMORPH \\
\hline $4.3-2.8-3.9$ & ECTOMORPHIC ENDOMORPH & $5.1-3.6-0.7$ & MESOMRPHIC ENDOMORPH \\
\hline $2.8-3.9-3.1$ & ECTOMORPHIC MESOMORPH & 7.6-4.8-0.1 & MESOMRPHIC ENDOMORPH \\
\hline $1.3-1.8-6.3$ & MESOMORPHIC ECTOMORPH & $9.4-8.4-0.1$ & MESOMRPHIC ENDOMORPH \\
\hline $6.1-5.5-0.8$ & MESOMORPHIC ENDOMORPH & $5.5-3.4-1.5$ & MESOMORPHIC ENDOMORPH \\
\hline $1.8-4.4-4.0$ & ECTOMORPHIC MESOMORPH & $3.9-3.2-3.1$ & MESOMORPHIC ENDOMORPH \\
\hline $5.3-3.6-3.0$ & MESOMORPHIC ENDOMORPH & $4.3-3.0-1.5$ & MESOMORPHIC ENDOMORPH \\
\hline $2.2-3.6-3.8$ & MESOMORPHIC ECTOMORPH & $3.8-2.2-3.4$ & ECTOMORPHIC ENDOMORPH \\
\hline 4.6-4.9-1.6 & & $6.7-3.8-1.3$ & MESOMORPHIC ENDOMORPH \\
\hline Percentage of & & Percentage of & \\
\hline Endomorphy & $50 \%$ & Endomorphy & $66.66 \%$ \\
\hline Mesomorphy & $21.43 \%$ & Mesomorphy & $0 \%$ \\
\hline Ectomorphy & $28.57 \%$ & Ectomorphy & $33.3 \%$ \\
\hline
\end{tabular}


From Table-4 we can determine the bodytypes of our experimental subjects' groups. From the above table it was found that half subjects of the male population were endomorphic as a whole \& on the other hand maximum $(67 \%)$ of the female population were endomorphic.

\section{Discussion}

The variable samples were based on 10 anthropometric parameters needed to determine somatotype characteristics using the Carter and Heath method [18]. The growth and development differences among the participants of these age group (10 -20 year) were very significant to compare them directly through anthropometric measurements, but when determining the somatotype using the Carter and Heath method[18], the only relevant interrelation was between 10 anthropometric parameters and chronological age. That fact nullified the age difference and enabled further comparisons. Fifty percent male subjects belonged to the group dominated by the endomorphic component, but in case of female subjects sixty seven percent had endomorphic component. The group with an accentuated influence of the endomorphic somatotype component but also a highly emphasised mesomorphic component was characterised by high levels of subcutaneous fat tissue. It included endomorphs, the majority of whom had an accentuated mesomorphic component (mesomorphic endomorphs), followed by those with a balance of the two components (mesomorphic-endomorphic); there were also a few ectomorphs with an accentuated endomorphic component.

The $21 \%$ of the male population and none of the female population belong to the group dominated by mesomorphic somatotype component. This group comprised subjects with high numerical values for the mesomorphic components compared to the other two somatotype components (endomorphic and ectomorphic). Based on the values of those two less emphasised components, it was possible to further divide the subjects of this group into those dominated by the endomorphic component of the mesomorphic somatotype (endomorphic mesomorphs), those dominated by the ectomorphic component (ectomorphic mesomorphs), and those with a balance of the two components (balanced mesomorphs).

The $29 \%$ of the males and $33 \%$ of the females belong to the group predominant ectomorphs dominated by the ectomorphic somatotype component. The values of the other two components (endomorphic and mesomorphic) were much less emphasised and subdivide this group into two ectomorphic subtypes (mesomorphic ectomorphs and balanced ectomorphs).

It seemed to be obvious that a mesomorphic predominance could play a decisive role in any sport, including Table Tennis [21, 22]. Indeed, several investigations carried out on Table Tennis players demonstrated a superior muscular development in lower extremities. But in our study it was found that only $21 \%$ of the males and none of the females were mesomorphic.

In a structurally complex game such as Table Tennis, competitive success is primarily a result of the quality and degree of technical and tactical knowledge. Accordingly, the differences in the competitive success of the young table tennis players are mostly the result of differing levels of technical as well as tactical skills. Those differences are much more salient at this competitive level than among top senior players where a high level of technical/tactical knowledge can be assumed.

Although the existence of suitable morphological features certainly represents an advantage and plays a role in achieving competitive success (especially among top players), in a technically complex game like Table Tennis, it is just one of many factors that influence competitive success. At this age, a player's basic anthropometric characteristics and body constitution is an important factor but far from crucial factor in achieving top competitive results.

The predominance of a physique dominated by the mesomorphic and ectomorphic somatotype components only reveals the potential advantage of these types of body constitution in increasing the likelihood of success, but it is not a decisive factor that directly influences competitive success among young table tennis players.

Average body fat percentage and average total fat content $(\mathrm{kg})$ were significantly $(\mathrm{p}<0.05)$ higher in female players as the female players possessed a high quantity of subcutaneous fat. On the other hand average lean body mass $(\mathrm{kg})$ of the male players was significantly $(\mathrm{p}<0.05)$ higher than their female counterparts as the quantity of subcutaneous fat for the males were comparatively low. Average elastic leg flexibility of the male players was significantly $(\mathrm{p}<0.05)$ higher than the female, so the leg flexibility of the male players was better than the female players. Average biepicondylar humerus and biepicondylar femur breadths were found significantly $(\mathrm{p}<0.05)$ higher in male than female as the bone breadth of the males were high.

\section{Conclusion}

In the present study male and female trained Table Tennis players were analyzed in different ways, like height, weight, body composition, anthropometric parameters, flexibility, elastic leg strength, hand span, handgrip strength and agility. All values were correlated with each other to assess the differences between male and female Table Tennis players in their musculoskeletal fitness and body type.

High correlation values were found between arm girth, calf girth, biepicondylar humerus breadth, biepicondylar femur breadth, chest circumference, waist circumference, hip circumference, mid thigh circumference and upper thigh circumference in both genders. It was found that there were only $21 \%$ of the males and none of the female players were mesomorphic. But mesomorphy was essential for the 
improvement of performance in table tennis players.

It could be concluded that the physical training procedure might not be specific to the event's demand. Musculoskeletal development of the players did not specify the Table Tennis event. Endomorphy had been found in both male and female players. Thus the muscle content of the players must be enhanced by improving the event specific physical training and by prescribing proper diet to the players. It is advisable to integrate educational and nutritional strategies in order to maintain an adequate body fat content.

\section{References}

[1] P. Faccini, M. Faina, E. Scarpellini and A. Dal Monte, "Il costo energetico nel tennistavolo", Scuola dello Sport, Vol. 8, No. 17 , pp. 38-42, 1989.

[2] Bagur JC, and Serra JR. "Clasificación de la actividad física y de los deportes más comunes en función del trabajo que requieren". In: Serra, J. R. Prescripción de ejercicio físico para la salud, Paidotribo, Barcelona, 1996.

[3] Bermejo JL, Quintano J, Ramos M, and Dongping Z. Tenis de mesa, Comité Olímpico Español, Madrid, 1991.

[4] Esparza F. Manual de cineantropometría, GREC-FEMEDE, Pamplona, 1993.

[5] Carter, J. E. L., \& Heath, B. H. (1990). Somatotyping Development and application. Cambridge: Cambridge University Press.

[6] Reilly T, Bangsbo J, Franks A. Anthropometric and physiological predispositions for elite soccer. Journal of Sports Sciences, 18, 2000, 669-683.

[7] Ackland TR, Ong KB, Kerr DA, Ridge B. Morphological characteristics of Olympic sprint canoe and kayak paddlers. Journal of Science and medicine in sports, 6(3), 2003, 285294.

[8] Gualdi-Russo, E., \& Zaccagni, L. (2001). Somatotype, role and performance in elite volleyball players. The Journal of Sports Medicine and Physical Fitness, 41 (2), 256-262.

[9] Siders, W. (1993). Relationships among swimming performance, body composition and somatotype in competitive collegiate swimmers. Journal of Sports Medicine and Physical Fitness, 33 (2), 166-171.
[10] Sullivan, J. (1994). Anthropometric characteristics and performance related predictors of success in adolescent pol. Journal of Sports Medicine, 34(2), 179-184.

[11] Sanchez-Munoz, C., Sanz, D., \& Zabala, M. (2007). Anthropometric characteristics, body composition and somatotype of elite junior tennis players. British Journal of Sports Medicine, 41(11), 793-799.

[12] De Hojo, M., Sanudo, B., \& Paris, F. (2009). Analysis of the somatotype, body composition and anthropometry in badminton players between 12 and 16 years. In: A. Lees, D. Cabello, G.

[13] Torres Luque (Eds.), Science and racket sports IV (pp.112117). London, New York: Routledge.

[14] Maud, P. J. and Foster, C. (1995) Physiological Assessment of Human Fitness. Champaign, Illinios: Human Kinetics.

[15] Mac Dougall, J. D.; Wenger, H.A. and Green, H.J. (1991) Physiological Testing of the High-Performance Athlete. Champaign, Illinios: Human Kinetics.

[16] International Society for the Advancement of Kinanthropometry (ISAK), available at www.isakonline.com.

[17] ISAK, International Standards for Anthropometric Assessment, ISAK manual, International Society for the Advancement of Kinanthropometry (ISAK), Lower Hutt, New Zealand, 2011.

[18] Heath, B. H., and Carter, J. E. L., A modified somatotype method, American journal of Physical Anthropology, 1967, 27: 57-74.

[19] Durnin, J. V. G. A., Womersly, J., Body fat assessed from total body density and its estimation from skinfold thicknesses, British Journal of Nutrition, 32: 77-79, 1974.

[20] Brozek, J., Grande, F., Anderson, J. T., Keys, A., Densiometric analysis of body composition : revision of some quantitative assumption, Annals of the New York Academy of Sciences, 110: 113-140, 1963.

[21] De Rose EH, and Guimaraes AC. "A model for optimization of somatotype in young athletes". In: Ostin M, Buenen G, Simons J: Kinanthropometry II, University Park Press, Baltimore, 1980.

[22] Pradas F, Carrasco L, Martínez E, and Herrero R. "Anthropometric profile, somatotype, and body composition of young table tennis players", Revista Internacional de Ciencias del Deporte, Vol. 3, No.7, pp. 11-23, 2007. 